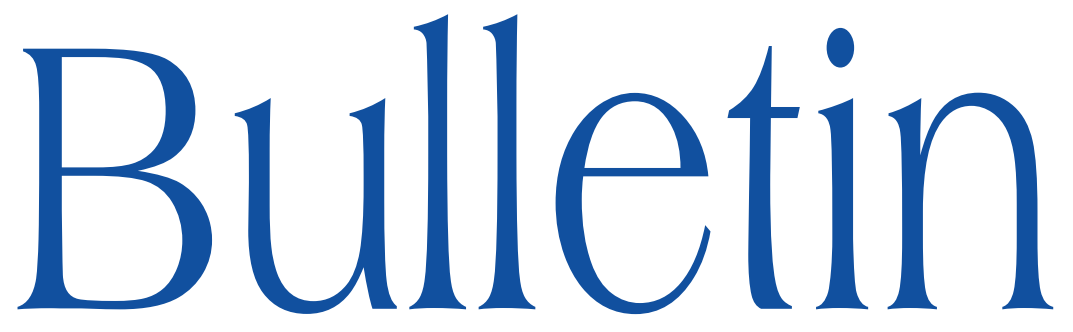

de la SOCIÉTÉ MATHÉMATIQUE DE FRANCE

\title{
WEYL FORMULA WITH OPTIMAL REMAINDER ESTIMATE OF SOME ELASTIC NETWORKS AND APPLICATIONS
}

Kaïs Ammari \& Mouez Dimassi

\section{Tome 138}

Fascicule 3 


\title{
WEYL FORMULA WITH OPTIMAL REMAINDER ESTIMATE OF SOME ELASTIC NETWORKS AND APPLICATIONS
}

\author{
BY KAÏs Ammari \& Mouez Dimassi
}

\begin{abstract}
We consider a network of vibrating elastic strings and Euler-Bernoulli beams. Using a generalized Poisson formula and some Tauberian theorem, we give a Weyl formula with optimal remainder estimate. As a consequence we prove some observability and stabilization results.

RÉSUmé (Formule de Weyl avec reste optimal de quelques réseaux élastiques et applications)

Nous considérons un réseau de cordes et de poutres d'Euler-Bernoulli. En utilisant une formule de Poisson généralisée et un théorème taubérien nous prouvons une formule de Weyl avec reste optimal. Comme conséquence nous prouvons des résultats d'observabilités et de stabilisations.
\end{abstract}

\section{Introduction}

In the last years various models of multiple-link flexible structures have been given and developed. The structures which we have in mind consist of finitely many interconnected flexible elements like strings, beams, plates representative

Texte reçu le 20 décembre 2007, révisé le 29 octobre 2008, accepté le 30 octobre 2008

Kaïs Ammari, Département de Mathématiques, Faculté des Sciences de Monastir, 5019

Monastir, Tunisie • E-mail : kais.ammari@fsm.rnu.tn

Mouez Dimassi, Département de Mathématiques, Institut Galilée, Université Paris 13, 99, avenue Jean-Baptiste Clément 93430 Villetaneuse, France

E-mail : dimassi@math.univ-paris13.fr

2000 Mathematics Subject Classification. - 35P20, 93D15, 93D20.

Key words and phrases. - Networks of strings, networks of Euler-Bernoulli beams, Tauberian theorem, Weyl formula. 
of trusses, frames, solar panels, antennae deformable mirrors, for more details concerning the models see [12]. "The spectral analysis of such models displays, in addition to its own mathematical interest, control and stabilization problems, see $[9,10,11,12],[15,16,18]$ and $[1,3,4,5]$.

First of all, we introduce some notations, which are simply those of [7], [14], we refer to [7] for more details, that is needed to formulate the problem under consideration.

Let $\Gamma$ be a connected topological graph embedded in $\mathbb{R}^{m}, m \in \mathbb{N}^{*}$, with $n$ verticies $\phi=\left\{E_{i}, 1 \leq i \leq n\right\}$ and $N$ edges $\mathscr{C}=\left\{k_{i}, 1 \leq i \leq N\right\}$. Each edge $k_{j}$ is a Jordan curve in $\mathbb{R}^{m}$ and is assumed to be parametrized by its arc length parameter $x_{j}$, such that the parametrizations

$$
\pi_{j}:\left[0, l_{j}\right] \rightarrow k_{j}: x_{j} \longmapsto \pi_{j}\left(x_{j}\right)
$$

is $C^{\nu}\left(\left[0, l_{j}\right], \mathbb{R}^{m}\right)$ for all $1 \leq j \leq N$.

We now define the $C^{\nu}$-network $G$ associated with $\Gamma$ as the union

$$
G=\cup_{j=1}^{N} k_{j} .
$$

The incidence matrix $D=\left(d_{i j}\right)_{n \times N}$ of $\Gamma$ is defined by

$$
d_{i j}=\left\{\begin{array}{l}
1 \text { if } \pi_{j}\left(l_{i}\right)=E_{i}, \\
-1 \text { if } \pi_{j}(0)=E_{i}, \\
0 \text { otherwise. }
\end{array}\right.
$$

The adjacency matrix $\mathcal{E}=\left(e_{i h}\right)_{n \times n}$ of $\Gamma$ is given by

$$
e_{i h}=\left\{\begin{array}{l}
1 \text { if there exists an edge } k_{s(i, h)} \text { between } E_{i} \text { and } E_{h} \\
0 \text { otherwise. }
\end{array}\right.
$$

The valence ${ }^{(*)}$ of the node $E_{i}$ will be noted $\gamma\left(E_{i}\right)$. There are two types of nodes: the interior nodes int $\phi=\left\{E_{i} \in \phi ; \gamma\left(E_{i}\right)>1\right\}$ and the boundary nodes $\partial \phi=\left\{E_{i} \in \&: \gamma\left(E_{i}\right)=1\right\}$. In the following we will denote $I_{\text {ext }}=$ $\left\{i \in\{1, \ldots, n\}: \gamma\left(E_{i}\right)=1\right\}$ and $I_{\text {int }}=\{1, \ldots, n\} \backslash I_{\text {ext }}$. We denote by $N_{i}=$ $\left\{j \in\{1, \ldots, n\}, E_{i} \in k_{j}\right\}$ the set of edges adjacent to $E_{i}$. We remark that if $E_{i} \in \partial \phi$, then $N_{i}$ is a singleton which is denoted by $\left\{j_{i}\right\}$.

For a function $u: G \rightarrow \mathbb{R}$, we set $u_{j}=u \circ \pi_{j}:\left[0, l_{j}\right] \rightarrow \mathbb{R}$, its restriction to the edge $k_{j}$. We further use the abbreviations:

$$
u_{j}\left(E_{i}\right)=u_{j}\left(\pi_{j}^{-1}\left(E_{i}\right)\right), u_{j x_{j}^{(n)}}\left(E_{i}\right)=\frac{d^{n} u_{j}}{d x_{j}^{n}}\left(\pi_{j}^{-1}\left(E_{i}\right)\right), n \in \mathbb{N}^{*} .
$$

Finally, differentiations are carried out on each edge $k_{j}$ with respect to the arc length parameter $x_{j}$.

(*) The valence of the node $E_{i}$ is the cardinal of the set of edges adjacent to $E_{i}$.

TOME $138-2010-\mathrm{N}^{\circ} 3$ 
We consider the following operator $\Delta_{G}$ on the Hilbert space $H=$ $\prod_{j=1}^{N} L^{2}\left(0, l_{j}\right)$, endowed with the usual product norm.

$$
\begin{aligned}
D\left(\Delta_{G}\right)= & \left\{u \in H, u_{j} \in H^{2}\left(0, l_{j}\right) \text { satisfying }(1.1)-(1.3)\right\} \\
& \Delta_{G} u=\left(-u_{j x_{j}^{(2)}}\right)_{j=1}^{N}, \forall u \in D\left(\Delta_{G}\right) .
\end{aligned}
$$

If $O=\left(O_{i h}\right)_{n \times n}$ is the orientation matrix defined by

$$
O_{i h}=\left\{\begin{array}{l}
1 \text { if } k_{s(i, h)} \text { is directed from } E_{i} \text { to } E_{h} \\
-1 \text { if } k_{s(i, h)} \text { is directed from } E_{h} \text { to } E_{i} \\
0 \text { else }
\end{array}\right.
$$

$$
\begin{gathered}
u \text { is continuous on } G, \\
\sum_{j=s(i, h) \in N_{i}} O_{i h} u_{j x_{j}}\left(E_{i}\right)=0, \forall i=1, \ldots, n, \\
u_{j_{i}}\left(E_{i}\right)=0, \forall i \in I_{e x t} .
\end{gathered}
$$

We study a model of networks of strings and of Euler-Bernoulli beams.

More precisely we consider the following initial problems:

on a finite network, of length $L$, made of edges $k_{j}$, identified to a real interval of length $l_{j}, j=1, \ldots, N$, (i.e. $L=\sum_{i=1}^{N} l_{i}$ ) we consider the eigenvalue problem

$$
-\frac{d^{2} u_{j}}{d x_{j}^{2}}=\lambda u_{j}, \quad k_{j}, j=1, \ldots, N
$$

$$
u \text { satisfies }(1.1)-(1.3)
$$

and

$$
\begin{gathered}
\frac{d^{4} u_{j}}{d x_{j}^{4}}=\lambda u_{j}, \quad k_{j}, j=1, \ldots, N, \\
O_{i h} u_{j x_{j}^{(2)}}\left(E_{i}\right)=O_{i k} u_{l x_{l}^{(2)}}\left(E_{i}\right), \text { if } j=s(i, h), l=s(i, k), \\
\sum_{j=s(i, h) \in N_{i}} O_{i h} u_{j x_{j}^{(3)}}\left(E_{i}\right)=0, \forall i=1, \ldots, n, \\
u_{j_{i}}\left(E_{i}\right)=0, u_{j_{i}, x_{j_{i}}^{(2)}}\left(E_{i}\right)=0, \forall i \in I_{e x t}, \\
u \text { satisfies }(1.1)-(1.2) .
\end{gathered}
$$


In the present paper we give some asymptotic Weyl formula of some networks of strings and of Euler-Bernoulli beams.

The plan of the paper is as follows. In the following section we give precise statements of the main results. The two last sections are devoted to some applications and related question.

\section{Asymptotics with optimal remainder estimates}

Let $\lambda_{0}<\lambda_{1} \leq \lambda_{2} \leq \cdots \leq \lambda_{n} \leq \cdots$ be the eigenvalues, repeated according to their multiplicity, of the self-adjoint operator $\Delta_{G}$ on a $C^{2}$-network $G$ which is defined in Section 1.

We introduce the counting function of eigenvalues

$$
\left.\left.N_{\Delta_{G}}(\lambda):=\# \sigma\left(\Delta_{G}\right) \cap\right]-\infty, \lambda\right],
$$

where in general \# $A$ denotes the number of elements of $A$.

Our main result can now be stated as follows.

TheOREM 2.1. - There exists $\lambda_{0} \gg 1$ such that

$$
N_{\Delta_{G}}(\lambda)=\frac{L}{\pi} \sqrt{\lambda}+\theta(1),
$$

uniformly on $\lambda \in] \lambda_{0},+\infty[$.

Proof. - Let $\chi \in C_{0}^{\infty}(]-\epsilon, \epsilon[)$, with $\chi(0)=1$. We choose $\epsilon>0$ small enough such that $l_{j}>\epsilon$ for all $j=1, \ldots, N$. We may chose $\chi$ with the additional property $\widehat{\chi}(t) \geq 0$ and $\widehat{\chi}(0)>0$. In fact, it suffices to choose $\chi=\psi \star \tilde{\psi}$ for a suitable $\psi \in C_{0}^{\infty}$. Here $\tilde{\psi}(t)=\psi(-t)$. We define

$$
\mu(\lambda)=\#\left\{j ; \mu_{j} \leq \lambda\right\}=\sum_{\mu_{j} \leq \lambda}(1)
$$

where $\mu_{j}=\sqrt{\lambda_{j}}$. According to Lemma 4.1 in the Appendix, we have

$$
\sum_{j=0}^{\infty} \widehat{\chi}\left(\lambda+\mu_{j}\right)+\sum_{j=0}^{\infty} \widehat{\chi}\left(\lambda-\mu_{j}\right)=2 L \chi(0)+(n-N) \widehat{\chi}(\lambda) .
$$

Since $\widehat{\chi}(0)>0$, there exists $\delta>0$ such that $\widehat{\chi}(t)>\frac{1}{2} \widehat{\chi}(0)$ for all $t \in[-\delta, \delta]$. Combining this with the fact that $\hat{\chi}(t) \geq 0$ for all $t \in \mathbb{R}$, and using (2.1), we obtain

$$
\begin{gathered}
\#\left\{j ; \mu_{j} \in[\lambda-\delta, \lambda+\delta]\right\} \leq \frac{2}{\widehat{\chi}(0)} \sum_{j}\left(\widehat{\chi}\left(\lambda+\mu_{j}\right)+\widehat{\chi}\left(\lambda-\mu_{j}\right)\right) \\
\leq \frac{2}{\widehat{\chi}(0)}(2 L \chi(0)+(n-N) \widehat{\chi}(\lambda))=\Theta(1),
\end{gathered}
$$

TOME $138-2010-\mathrm{N}^{\mathrm{O}} 3$ 
uniformly on $\lambda \in \mathbb{R}$. Without any loss of generality, we may assume that $\delta=1$. Writing

$$
\mu(\lambda) \leq \sum_{0}^{[\lambda]} \mu(j+1)-\mu(j),
$$

with $[\lambda]=$ largest integer $\leq \lambda$, we obtain

$$
\mu(\lambda)=\theta(\lambda)
$$

which yields

$$
\mu_{j}=\Theta(1) j .
$$

An immediate consequence of the above equality is that

$$
\begin{aligned}
& \sum_{j=0}^{\infty} \widehat{\chi}\left(\lambda-\mu_{j}\right)=\theta\left(\langle\lambda\rangle^{-\infty}\right), \text { for } \lambda<0, \\
& \sum_{j=0}^{\infty} \widehat{\chi}\left(\lambda+\mu_{j}\right)=\theta\left(\langle\lambda\rangle^{-\infty}\right), \text { for } \lambda>0 .
\end{aligned}
$$

Here $\langle\lambda\rangle=\left(1+|\lambda|^{2}\right)^{\frac{1}{2}}$. Combining this with (2.1), we obtain

$$
\sum_{j=0}^{\infty} \widehat{\chi}\left(\lambda-\mu_{j}\right)=2 L \chi(0)+\theta\left(\langle\lambda\rangle^{-\infty}\right), \text { for } \lambda>0 .
$$

Put $K(\lambda)=\int_{-\infty}^{\lambda} \widehat{\chi}(\tau) d \tau$. We have

$$
\begin{gathered}
\int K(\lambda-x) d \mu(x)=\sum K\left(\lambda-\mu_{n}\right) \\
=\sum \int_{-\infty}^{\lambda-\mu_{n}} \widehat{\chi}(\tau) d \tau=\sum \int_{-\infty}^{\lambda} \widehat{\chi}\left(x-\mu_{n}\right) d x .
\end{gathered}
$$

We recall that $\mu(\lambda)=\sum_{\mu_{n} \leq \lambda}(1)$. The estimates (2.4) and (2.5) yield

$$
\begin{gathered}
\int K(\lambda-x) d \mu(x)=2 L \chi(0) \int_{0}^{\lambda} d x+\Theta(1) \\
=2 L \chi(0) \lambda+\Theta(1), \lambda \rightarrow+\infty
\end{gathered}
$$

We rewrite the left hand side of (2.6) as

$$
\begin{aligned}
& \int K(\lambda-x) d \mu(x)=\int \mu(\lambda-\tau) \widehat{\chi}(\tau) d \tau \\
= & \int(\mu(\lambda-\tau)-\mu(\lambda)) \widehat{\chi}(\tau) d \tau+2 \pi \mu(\lambda),
\end{aligned}
$$


since $\int \widehat{\chi}(\tau) d \tau=2 \pi \chi(0)=2 \pi$. For $\lambda \gg 1$, we get from (2.2)

$$
|\mu(\lambda-\tau)-\mu(\lambda)| \leq C(1+|\tau|) .
$$

Consequently,

$$
\int(\mu(\lambda-\tau)-\mu(\lambda)) \widehat{\chi}(\tau) d \tau=\Theta(1),(\lambda \rightarrow+\infty) .
$$

Putting together (2.7), (2.8) and (2.9), we get

$$
\mu(\lambda)=\frac{L}{\pi} \lambda+\Theta(1) .
$$

Combining this with the fact that $\mu(\sqrt{\lambda})=N_{\Delta_{G}}(\lambda)$, we get Theorem 2.1.

As a consequence we have the following result concerning the beams networks:

COROLlary 2.2. - There exists $\lambda_{0} \gg 1$ such that

$$
\left.\left.N_{\Delta_{G}^{2}}(\lambda):=\# \sigma\left(\Delta_{G}^{2}\right) \cap\right]-\infty, \lambda\right]=\frac{L}{\pi} \lambda+\theta(1),
$$

uniformly on $\lambda \in] \lambda_{0},+\infty\left[\right.$ and where $D\left(\Delta_{G}^{2}\right)=\left\{u \in H, u_{j} \in H^{4}\left(0, l_{j}\right)\right.$ satisfying $(1.1)-(1.2)$ and $(1.7)-(1.8)\}$.

REMARK 2.3. - We remark that our method is valid for all elliptic operator in a graph.

As consequence of Theorem 2.1 we have the following result:

Corollary 2.4. - There exist $M \in \mathbb{N}^{*}$ and $\eta>0$ such that

$$
\mu_{n+M}-\mu_{n} \geq \eta M, \forall n \geq 0
$$

where $\mu_{k}=\sqrt{\lambda_{k}}, \forall k \geq 0$.

Proof. - From Theorem 2.1, there exists $n_{0} \in \mathbb{N}^{*}$ and $C>0$ such that

$$
-C \leq \mu_{n}-\frac{\pi}{L} n \leq C,
$$

for all $n \geq n_{0}$. Changing $C$ by $\tilde{C}>C$, we may assume that $n_{0}=0$. Using the above inequality, we obtain

$$
\frac{\pi}{L} M-2 \tilde{C} \leq \mu_{n+M}-\mu_{n}
$$

for all $n \geq 0, M \in \mathbb{N}^{*}$. Choosing $M$ large enough so that $\frac{\pi}{L} M-2 \tilde{C}>\eta M$ we obtain the result. 


\section{Applications}

Let $H$ be a Hilbert space equipped with the norm $\|\cdot\|_{H}$, and let $A_{1}: \mathscr{D}\left(A_{1}\right) \rightarrow$ $H$ be a self-adjoint, positive and boundedly invertible operator with compact resolvent. We introduce the scale of Hilbert spaces $H_{\alpha}, \alpha \in \mathbb{R}$, as follows: for every $\alpha \geq 0, H_{\alpha}=\mathscr{D}\left(A_{1}^{\alpha}\right)$, with the norm $\|z\|_{\alpha}=\left\|A_{1}^{\alpha} z\right\|_{H}$. The space $H_{-\alpha}$ is defined by duality with respect to the pivot space $H$ a follows: $H_{-\alpha}=H_{\alpha}^{*}$ for $\alpha>0$. The operator $A_{1}$ can be extended (or restricted) to each $H_{\alpha}$, such that it becomes a bounded operator

$$
A_{1}: H_{\alpha} \rightarrow H_{\alpha-1} \quad \forall \alpha \in \mathbb{R} .
$$

The second ingredient needed for our construction is a bounded linear operator $B_{1}: U \rightarrow H_{-\frac{1}{2}}$, where $U$ is another Hilbert space which will be identified with its dual. The systems we consider are described by

$$
\begin{aligned}
\ddot{w}(t)+A_{1} w(t)+B_{1} y(t) & =0, w(0)=w_{0}, \dot{w}(0)=w_{1}, \\
y(t) & =B_{1}^{*} \dot{w}(t),
\end{aligned}
$$

where $t \in[0, \infty)$ is the time. The equation (3.11) is understood as an equation in $H_{-\frac{1}{2}}$, i.e., all the terms are in $H_{-\frac{1}{2}}$. Most of the linear equations modelling the damped vibrations of elastic structures can be written in the form (3.11), where $w$ stands for the displacement field and the term $B_{1} B_{1}^{*} \dot{w}(t)$, represents a viscous feedback damping. The system (3.11)-(3.12) is well-posed:

For $\left(w_{0}, w_{1}\right) \in H_{\frac{1}{2}} \times H$, the problem (3.11)-(3.12) admits a unique solution

$$
w \in C\left([0, \infty) ; H_{\frac{1}{2}}\right) \cap C^{1}([0, \infty) ; H)
$$

such that $B_{1}^{*} w(\cdot) \in H^{1}(0, T ; U)$. Moreover $w$ satisfies, for all $t \geq 0$, the energy estimate

$$
\left\|\left(w_{0}, w_{1}\right)\right\|_{H_{\frac{1}{2}} \times H}^{2}-\|(w(t), \dot{w}(t))\|_{H_{\frac{1}{2}} \times H}^{2}=2 \int_{0}^{t}\left\|\frac{\mathrm{d}}{\mathrm{d} t} B_{1}^{*} w(s)\right\|_{U}^{2} \mathrm{~d} s .
$$

From (3.13) it follows that the mapping $t \mapsto\|(w(t), \dot{w}(t))\|_{H_{\frac{1}{2}} \times H}^{2}$ is non increasing.

We consider the initial value problem

$$
\begin{gathered}
\ddot{\varphi}(t)+A_{1} \varphi(t)=0, \\
\varphi(0)=w_{0}, \dot{\varphi}(0)=w_{1} .
\end{gathered}
$$

It is well known that (3.14)-(3.15) is well-posed in $H_{1} \times H_{\frac{1}{2}}$ and in $H_{\frac{1}{2}} \times H$.

Consider now the unbounded linear operator

$$
\mathscr{G}_{d}: \mathscr{D}\left(\mathscr{G}_{d}\right) \rightarrow H_{\frac{1}{2}} \times H, \mathscr{G}_{d}=\left(\begin{array}{cc}
0 & I \\
-A_{1}-B_{1} B_{1}^{*}
\end{array}\right),
$$


where

$$
\mathscr{D}\left(\mathscr{G}_{d}\right)=\left\{(u, v) \in H_{\frac{1}{2}} \times H, A_{1} u+B_{1} B_{1}^{*} v \in H, v \in H_{\frac{1}{2}}\right\} .
$$

The result below, see [6], shows that, under a certain regularity assumption, the polynomial stability of (3.11)-(3.12) is a consequence of a weak observability inequality. More precisely, we have:

THEOREM 3.1. - Assume that for any $\gamma>0$ we have

$$
\sup _{\operatorname{Re} \lambda=\gamma}\left\|\lambda B_{1}^{*}\left(\lambda^{2} I+A_{1}\right)^{-1} B_{1}\right\|_{\mathscr{L}(U)}<\infty .
$$

Then the following assertion holds true:

If there exist $T, C>0, \alpha>-\frac{1}{2}$ such that: $\forall\left(w_{0}, w_{1}\right) \in H_{1} \times H_{\frac{1}{2}}$ we have

$$
\left\|B_{1}^{*} \varphi^{\prime}(t)\right\|_{L^{2}(0, T ; U)} \geq C\left\|\left(w_{0}, w_{1}\right)\right\|_{H_{-\alpha} \times H_{-\alpha-\frac{1}{2}}},
$$

where $\varphi(t)$ is a solution of (3.14)-(3.15).

Then there exists a constant $C_{1}>0$ such that for all $t>0$ and for all $\left(w^{0}, w^{1}\right) \in \mathscr{D}\left(\mathscr{G}_{d}\right)$ we have

$$
\|(w(t), \dot{w}(t))\|_{H_{\frac{1}{2}} \times H} \leq \frac{C_{1}}{(1+t)^{\frac{1}{4 \alpha+2}}}\left\|\left(w^{0}, w^{1}\right)\right\|_{\mathscr{D}\left(\mathscr{C}_{d}\right)} .
$$

\subsection{Application to a polynomial stabilization of a star-shaped network of strings.}

- We consider the following initial and boundary value problems :

$$
\begin{gathered}
\frac{\partial^{2} u_{i}}{\partial t^{2}}(x, t)-\frac{\partial^{2} u_{i}}{\partial x^{2}}(x, t)=0, \quad 0<x<l_{i}, t>0, \\
u_{i}\left(l_{i}, t\right)=0, \quad t>0, \\
u_{i}(0, t)=u_{j}(0, t), \quad t>0, \\
\sum_{i=1}^{N} \frac{\partial u_{i}}{\partial x}(0, t)=\frac{\partial u_{1}}{\partial t}(0, t), \quad t>0, \\
u_{i}(x, 0)=u_{i}^{0}(x), \frac{\partial u_{i}}{\partial t}(x, 0)=u_{i}^{1}(x), \quad 0<x<l_{i},
\end{gathered}
$$

for $i, j=1, \ldots, N$ and where $u_{i}:\left[0, l_{i}\right] \times(0,+\infty) \rightarrow \mathbb{R}, i=1, \ldots, N, N \geq 2$ be the displacement of the string of length $l_{i}$. Denote by $L=\sum_{i=1}^{N} l_{i}$. 
Let $H=\prod_{i=1}^{N} L^{2}\left(0, l_{i}\right), A_{1}=-\frac{d^{2}}{d x^{2}}$

$$
\begin{gathered}
\mathscr{D}\left(A_{1}\right)=\left\{\left(\left(u_{i}\right)_{i=1, \ldots, N}\right) \in \prod_{i=1}^{N} H^{2}\left(0, l_{i}\right), u_{i}(0)=u_{j}(0), \forall i, j=1, \ldots, N,\right. \\
\left.u_{i}\left(l_{i}\right)=0, \sum_{i=1}^{N} \frac{d u_{i}}{d x}(0)=0\right\} .
\end{gathered}
$$

$\mathscr{D}\left(A_{1}^{\frac{1}{2}}\right)$ is the completed of $\mathscr{D}\left(A_{1}\right)$ with respect to the norm

$$
\|z\|_{\mathscr{D}\left(A_{1}^{\frac{1}{2}}\right)}=\sqrt{\left(A_{1} z, z\right)_{H}}=\sqrt{\sum_{i=1}^{N} \int_{0}^{l_{i}}-\frac{d^{2} z_{i}}{d x^{2}} z_{i} d x .}
$$

Let $B_{1} \in \mathscr{L}\left(\mathbb{R}, \mathscr{D}\left(A_{1}^{\frac{1}{2}}\right)^{\prime}\right), B_{1} v=\left(A_{1}\right)_{-1} \mathcal{N} v, \forall v \in \mathbb{R}$, where $\left(A_{1}\right)_{-1}: \mathscr{D}\left(A_{1}^{\frac{1}{2}}\right) \rightarrow$ $\mathscr{D}\left(A_{1}^{\frac{1}{2}}\right)^{\prime}$ is an extension of $A_{1}$ to $\mathscr{D}\left(A_{1}^{\frac{1}{2}}\right), \mathcal{N} \in \mathcal{L}\left(\mathbb{R}, \mathscr{D}\left(A_{1}^{\frac{1}{2}}\right)\right)$ and $\mathcal{N} v$ is a solution of:

$$
\begin{gathered}
\frac{d^{2}(\mathcal{N} v)_{i}}{d x^{2}}=0, \quad 0<x<l_{i}, \\
(\mathcal{N} v)_{i}\left(l_{i}\right)=0 \\
(\mathcal{N} v)_{i}(0)=(\mathcal{N} v)_{j}(0) \\
\sum_{i=1}^{N} \frac{d(\mathcal{N} v)_{i}}{d x}(0)=v
\end{gathered}
$$

for all $i, j=1, \ldots, N$, and

$$
B_{1}^{*} \psi=\psi_{1}(0), \forall \psi \in \mathscr{D}\left(A_{1}^{\frac{1}{2}}\right) .
$$

We denote by $\lambda_{k}=\mu_{k}^{2}$ the eigenvalues of $A_{1}$. In the case: $\frac{l_{i}}{l_{j}} \notin \mathbb{Q}, \forall 1 \leq i \neq j \leq$ $N$, the eigenvalues $\lambda_{k}$ are simple (see [3]) and the corresponding eigenfunctions are given by:

$$
\phi_{k}^{i}(x)=\frac{\sin \left(\mu_{k}\left(x-l_{i}\right)\right)}{\sin \left(\mu_{k} l_{i}\right)\left(\sum_{i=1}^{N} \frac{l_{i}}{\sin ^{2}\left(\mu_{k} l_{i}\right)}\right)^{\frac{1}{2}}}, i=1, \ldots, N .
$$

We define the energy of $u_{i}, i=1, \ldots, N$ of (3.20)-(3.24) at instant $t$ by

$$
E(t)=\sum_{i=1}^{N} \frac{1}{2} \int_{0}^{l_{i}}\left(\left|\frac{\partial u_{i}}{\partial t}(x, t)\right|^{2}+\left|\frac{\partial u_{i}}{\partial x}(x, t)\right|^{2}\right) d x .
$$


The wellposedness space for (3.20)-(3.24) is $E=\mathscr{D}\left(A_{1}^{\frac{1}{2}}\right) \times \prod_{i=1}^{N} L^{2}\left(0, l_{i}\right)$. Denote $\mathscr{D}\left(\mathscr{G}_{d}\right)=\left\{\left(\left(u_{i}\right)_{i=1, \ldots, N},\left(v_{i}\right)_{i=1, \ldots, N}\right) \in\left[\mathscr{D}\left(A_{1}^{\frac{1}{2}}\right) \cap \prod_{i=1}^{N} H^{2}\left(0, l_{i}\right)\right] \times \mathscr{D}\left(A_{1}^{\frac{1}{2}}\right)\right.$

$$
\left.\sum_{i=1}^{N} \frac{d u_{i}}{d x}(0)=v_{1}(0)\right\}
$$

The corresponding operator $\mathscr{G}_{d}$ is defined by

$$
\mathscr{G}_{d}\left(\begin{array}{c}
u_{1} \\
\ldots \\
u_{N} \\
v_{1} \\
\cdots \\
v_{N}
\end{array}\right)=\left(\begin{array}{c}
v_{1} \\
\ldots \\
v_{N} \\
\frac{d^{2} u_{1}}{d x^{2}} \\
\ldots \\
\frac{d^{2} u_{N}}{d x^{2}}
\end{array}\right), \forall(u, v) \in \mathscr{D}\left(\mathscr{G}_{d}\right) .
$$

If $\left(u^{0}, u^{1}\right) \in E$, then the problem (3.20)-(3.24) admits a unique solution

$$
u \in C\left(0,+\infty ; \mathscr{D}\left(A_{1}^{\frac{1}{2}}\right)\right) \cap C^{1}\left(0,+\infty ; \prod_{i=1}^{N} L^{2}\left(0, l_{i}\right)\right)
$$

and we have: $\lim _{t \rightarrow+\infty} E(t)=0$ holds true for any finite energy solution of (3.20)(3.24) if and only if

$$
\frac{l_{i}}{l_{j}} \notin \mathbb{Q}, \forall 1 \leq i \neq j \leq N
$$

where $\mathbb{Q}$ is the set of all rational numbers.

Denote by $\&$ the set of all numbers $\rho$ such that $\rho \notin \mathbb{Q}$ and if $\left[0, a_{1}, \ldots, a_{n}, \ldots\right]$ is the expansion of $\rho$ as a continued fraction, then $\left(a_{n}\right)$ is bounded. Let us notice that $\&$ is obviously uncountable and, by classical results on diophantine approximation, its Lebesgue measure is equal to zero. Roughly speaking the set $\&$ contains the irrationals which are approximable by rational numbers. In particular, by Euler-Lagrange theorem $\phi$ contains all $l_{i} / l_{j}, 1 \leq i \neq j \leq N$ such that $l_{i} / l_{j}$ is an irrational quadratic number (i.e. satisfying a second degree equation with rational coefficients). According to [13], we have that $l_{i} / l_{j} \in$ $\phi, 1 \leq i \neq j \leq N$, if and only if there exists a positive constant $C$ such that: $\left|\left\|\left|\frac{l_{i}}{l_{j}} m\right|\right\|:=\min _{\frac{l_{i}}{l_{j}} m-x \in \mathbb{Z}}\right| x \mid \geq \frac{C}{m}, \forall m \in \mathbb{N}^{*}$.

TOME $138-2010-\mathrm{N}^{\mathrm{O}} 3$ 
Corollary 3.2. - 1. If $l_{i} / l_{j} \in \phi, \forall 1 \leq i \neq j \leq N$, there exists $\beta>0$ such that for all $t \geq 0$ we have

$$
E(t) \leq \frac{C}{(t+1)^{\frac{1}{\beta}}}\left\|\left(u^{0}, u^{1}\right)\right\|_{\mathscr{D}\left(\mathscr{G}_{d}\right)}^{2}, \quad \forall\left(u^{0}, u^{1}\right) \in \mathscr{D}\left(\mathscr{G}_{d}\right),
$$

where $C>0$ is a constant depending only on $l_{i}, i=1, \ldots, N$.

2. For all $\varepsilon>0$ there exists a set $B_{\varepsilon} \subset \mathbb{R}$, such that the Lebesgue measure of $\mathbb{R} \backslash B_{\varepsilon}$ is equal to zero, and a constants $\beta, C_{\varepsilon}>0$ for which, if $l_{i} / l_{j} \in$ $B_{\varepsilon}, 1 \leq i \neq j \leq N$, then for all $t \geq 0$

$$
E(t) \leq \frac{C_{\epsilon}}{(t+1)^{\frac{1}{\beta+\varepsilon}}}\left\|\left(u^{0}, u^{1}\right)\right\|_{\mathscr{D}\left(\mathscr{G}_{d}\right)}^{2}, \quad \forall\left(u^{0}, u^{1}\right) \in \mathscr{D}\left(\mathscr{G}_{d}\right),
$$

where $C_{\varepsilon}>0$ is a constant depending only on $l_{i}, i=1, \ldots, N$ and $\varepsilon$.

Proof. By a simple calculations we show that

$$
\lambda B_{1}^{*}\left(\lambda^{2} I+A_{1}\right)^{-1} B_{1}=-\left(\sum_{i=1}^{N} \operatorname{coth}\left(\lambda l_{i}\right)\right)^{-1}, \forall \lambda \in \mathbb{C}, \operatorname{Re} \lambda>0 .
$$

So the condition (3.17) is satisfied according to the following lemma:

Lemma 3.3. - [3, Lemma 3.3]

Let $\gamma>0$ be a fixed real number and $C_{\gamma}=\{\lambda \in \mathbb{C} \mid \operatorname{Re}(\lambda)=\gamma\}$. Then, the function

$$
f(\lambda)=\frac{1}{\sum_{i=1}^{N} \operatorname{coth}\left(\lambda l_{i}\right)}
$$

is bounded on $C_{\gamma}$.

For $k<0$, we denote by $\mu_{k}=-\mu_{-k}$. Let $0<\eta^{\prime} \leq \eta$ with $\eta^{\prime} \leq \frac{2 \mu_{1}}{M}$. We claim that

$$
\mu_{k+M}-\mu_{k} \geq \eta^{\prime} M, \forall k \in \mathbb{Z} \text {. }
$$

In fact, for $k>0$ resp. $(k+M<0)$ (3.35) follows from Corollary 2.4 resp.( Corollary 2.4 and the fact that $\left.\mu_{k}=-\mu_{-k}\right)$. For $k+M>0$ and $k<0$ we use that $\mu_{k+M}-\mu_{k}=\mu_{k+M}+\mu_{-k} \geq 2 \mu_{1} \geq M \eta^{\prime}$.

We denote by $A_{j}, j=1, \ldots, M$ the set of integers $m$ satisfying :

$$
\begin{gathered}
\mu_{m}-\mu_{m-1} \geq \eta^{\prime} \\
\mu_{n}-\mu_{n-1}<\eta^{\prime}, \forall m+1 \leq n \leq m+j-1 \\
\mu_{m+j}-\mu_{m+j-1} \geq \eta^{\prime} .
\end{gathered}
$$

Then the $M(M+1) / 2$ sets $A_{j}+k=\left\{n+k ; n \in A_{j}\right\}, 0 \leq k<j \leq M$ are disjoint and form a partition of the set $\mathbb{Z}$. Let us introduce for $m \in A_{j}$ the 
divided differences $e_{m}(t), \ldots, e_{m+j-1}(t)$ of the exponential functions $e^{i \mu_{n} t}, n=$ $m, \ldots, m+j-1$. Since $\mu_{k}$ are simple (see [3]) then $e_{k}$ (see [11]) is given by the following expression

$$
e_{k}(t)=\sum_{p=m}^{k}\left[\prod_{q=m, q \neq p}^{k}\left(\mu_{p}-\mu_{q}\right)\right]^{-1} e^{i \mu_{p} t}, \forall k=m, \ldots, m+j-1 .
$$

For

$$
\begin{gathered}
\left(u_{1}^{0}, \ldots, u_{N}^{0}, u_{1}^{1}, \ldots, u_{N}^{1}\right)^{t}= \\
\sum_{k \in \mathbb{Z}} a_{k}\left(\frac{1}{\mu_{k}} \phi_{k}^{1}, \ldots, \frac{1}{\mu_{k}} \phi_{k}^{N}, \phi_{k}^{1}, \ldots, \phi_{k}^{N}\right)^{t},\left(a_{k}\right)_{k \in \mathbb{Z}} \in l^{2},
\end{gathered}
$$

we have

$$
\frac{\partial \varphi_{1}}{\partial t}(0, t)=\sum_{k \in \mathbb{Z}} a_{k} e^{i \mu_{k} t} \phi_{k}^{1}(0)=\sum_{k \in \mathbb{Z}} b_{k} e_{k}(t),
$$

where $\varphi=\left(\varphi_{1}, \ldots, \varphi_{N}\right)^{t}$ is a solution of conservative system associated to (3.20)-(3.24). According to [11, Theorem 9.4] we have that for $T>\frac{2 \pi}{\eta}$ there exists a constant $C_{1}>0$ such that

$$
\int_{0}^{T}\left|\frac{\partial \varphi_{1}}{\partial t}(0, t)\right|^{2} d t \geq C_{1} \sum_{k \in \mathbb{Z}}\left|b_{k}\right|^{2}
$$

and if $l_{i} / l_{j} \in \phi, \forall 1 \leq i \neq j \leq N$, there exist $\beta, C_{2}>0$ such that we have:

$$
\int_{0}^{T}\left|\frac{\partial \varphi_{1}}{\partial t}(0, t)\right|^{2} d t \geq C_{2} \sum_{k \in \mathbb{Z}}\left|\mu_{k}\right|^{-\beta}\left|a_{k}\right|^{2} .
$$

Which implies, according to Theorem 3.1, the estimate (3.32).

In order to prove (3.33) we use a well-known result (see [8] p. 120) asserting that for all $\varepsilon>0$, there exists a set $B_{\varepsilon} \subset \mathbb{R}$, such that the Lebesgue measure of $\mathbb{R} \backslash B_{\varepsilon}$ is equal to zero, and a constant $C_{\varepsilon}>0$ for which, if $\xi \in B_{\varepsilon}$, then $\|\xi m\| \geq \frac{C_{\varepsilon}}{m^{1+\varepsilon}}$.

Let us notice that by Roth's theorem (see [17]) $B_{\varepsilon}$ contains all real numbers having the property that $\frac{l_{i}}{l_{j}}$ is an algebraic irrational (see [8] for details). If $l_{i} / l_{j} \in B_{\varepsilon}, 1 \leq i \neq j \leq N$, there exists a constant $C>0$ such that $\left\|m l_{i} / l_{j}\right\| \geq$ $\frac{C}{m^{1+\varepsilon}}, \forall m \geq 1$. Then, as above we have for $T>\frac{2 \pi}{\eta}$ that there exists a constant $C_{3}>0$ such that:

$$
\int_{0}^{T}\left|\frac{\partial \varphi_{1}}{\partial t}(0, t)\right|^{2} d t \geq C_{3} \sum_{k \in \mathbb{Z}}\left|\mu_{k}\right|^{-\beta-\varepsilon}\left|a_{k}\right|^{2}
$$

TOME $138-2010-\mathrm{N}^{\mathrm{O}} 3$ 
Which implies (3.33), according to Theorem 3.1.

\subsection{Application to a polynomial stabilization of a star-shaped network of Euler-}

Bernoulli beams. - We consider the following initial and boundary value problem :

$$
\begin{gathered}
\frac{\partial^{2} u_{i}}{\partial t^{2}}(x, t)+\frac{\partial^{4} u_{i}}{\partial x^{4}}(x, t)=0, \quad 0<x<l_{i}, t>0 \\
u_{i}\left(l_{i}, t\right)=0, \frac{\partial^{2} u_{i}}{\partial x^{2}}\left(l_{i}, t\right)=0, \quad t>0 \\
u_{i}(0, t)=u_{j}(0, t), \sum_{i=1}^{N} \frac{\partial u_{i}}{\partial x}(0, t)=0, \frac{\partial^{2} u_{i}}{\partial x^{2}}(0, t)=\frac{\partial^{2} u_{j}}{\partial x^{2}}(0, t), \quad t>0 \\
\sum_{i=1}^{N} \frac{\partial^{3} u_{i}}{\partial x^{3}}(0, t)=-\frac{\partial u_{1}}{\partial t}(0, t), \quad t>0, \\
u_{i}(x, 0)=u_{i}^{0}(x), \frac{\partial u_{i}}{\partial t}(x, 0)=u_{i}^{1}(x), \quad 0<x<l_{i},
\end{gathered}
$$

for $i, j=1, \ldots, N, 2 \leq N \in \mathbb{N}$ and where $u_{i}:\left[0, l_{i}\right] \times(0,+\infty) \rightarrow \mathbb{R}$, be the displacement of the beam of length $l_{i}$.

$$
\begin{aligned}
& \text { Let } H=\prod_{i=1}^{N} L^{2}\left(0, l_{i}\right), A_{1}=\frac{d^{4}}{d x^{4}}, \\
& \mathscr{D}\left(A_{1}\right)=\left\{\left(\left(u_{i}\right)_{i=1, \ldots, N}\right) \in \prod_{i=1}^{N} H^{4}\left(0, l_{i}\right), u_{i}(0)=u_{j}(0), \frac{d^{2} u_{i}}{d x^{2}}\left(l_{i}\right)=0,\right. \\
& \left.\frac{d u_{i}}{d x}(0)=\frac{d u_{j}}{d x}(0), \forall 1 \leq i, j \leq N, u_{i}\left(l_{i}\right)=0, \sum_{i=1}^{N} \frac{d^{3} u_{i}}{d x^{3}}(0)=0\right\}
\end{aligned}
$$

$\mathscr{D}\left(A_{1}^{\frac{1}{2}}\right)$ is the completed of $\mathscr{D}\left(A_{1}\right)$ with respect to the norm

$$
\|z\|_{\mathscr{D}\left(A_{1}^{\frac{1}{2}}\right)}=\sqrt{\left(A_{1} z, z\right)_{H}}=\sqrt{\sum_{i=1}^{N} \int_{0}^{l_{i}} \frac{d^{4} z_{i}}{d x^{4}} z_{i} d x .}
$$

Let $B_{1} \in \mathscr{L}\left(\mathbb{R}, \mathscr{D}\left(A_{1}^{\frac{1}{2}}\right)^{\prime}\right), B_{1} v=\left(A_{1}\right)_{-1} \mathcal{N} v, \forall v \in \mathbb{R}$, where $\left(A_{1}\right)_{-1}: \mathscr{D}\left(A_{1}^{\frac{1}{2}}\right) \rightarrow$ $\mathscr{D}\left(A_{1}^{\frac{1}{2}}\right)^{\prime}$ is an extension of $A_{1}$ to $\mathscr{D}\left(A_{1}^{\frac{1}{2}}\right), \mathcal{N} \in \mathcal{L}\left(\mathbb{R}, \mathscr{D}\left(A_{1}^{\frac{1}{2}}\right)\right)$ and $\mathcal{N} v$ is a solution of:

$$
\frac{d^{4}(\mathcal{N} v)_{i}}{d x^{4}}=0, \quad 0<x<l_{i},
$$




$$
\begin{gathered}
(\mathcal{N} v)_{i}\left(l_{i}\right)=0, \frac{d^{2}(\mathcal{N} v)_{i}}{d x^{2}}\left(l_{i}\right)=0 \\
(\mathcal{N} v)_{i}(0)=(\mathcal{N} v)_{j}(0), \frac{d(\mathcal{N} v)_{i}}{d x}(0)=\frac{d(\mathcal{N} v)_{j}}{d x}(0), \\
\sum_{i=1}^{N} \frac{d^{3}(\mathcal{N} v)_{i}}{d x^{3}}(0)=v
\end{gathered}
$$

for all $i, j=1, \ldots, N$, and

$$
B_{1}^{*} \psi=\psi_{1}(0), \forall \psi \in \mathscr{D}\left(A_{1}^{\frac{1}{2}}\right) .
$$

We denote by $\lambda_{k}=\mu_{k}^{4}$ the eigenvalues of $A_{1}$. In the case: $\frac{l_{i}}{l_{j}} \notin \mathbb{Q}, \forall 1 \leq i \neq j \leq$ $N$, the eigenvalues $\lambda_{k}$ are simple (see [1]) and the corresponding eigenfunctions are given by:

$$
\phi_{k}^{i}(x)=\frac{\sin \left(\mu_{k}\left(x-l_{i}\right)\right)}{\sin \left(\mu_{k} l_{i}\right)\left(\sum_{i=1}^{N} \frac{l_{i}}{\sin ^{2}\left(\mu_{k} l_{i}\right)}\right)^{\frac{1}{2}}}, i=1, \ldots, N .
$$

We define the energy of $u$ solution of (3.36)-(3.40) at instant $t$ by

$$
E(t)=\sum_{i=1}^{N} \frac{1}{2} \int_{0}^{l_{i}}\left(\left|\frac{\partial u_{i}}{\partial t}(x, t)\right|^{2}+\left|\frac{\partial^{2} u_{i}}{\partial x^{2}}(x, t)\right|^{2}\right) d x
$$

The wellposedness space for (3.36)-(3.40) is $X=\mathscr{D}\left(A_{1}^{\frac{1}{2}}\right) \times \prod_{i=1}^{N} L^{2}\left(0, l_{i}\right)$.

Denote

$$
\begin{aligned}
& \mathscr{D}\left(\mathscr{G}_{d}\right)=\left\{(u, v) \in\left[\mathscr{D}\left(A_{1}^{\frac{1}{2}}\right) \cap \prod_{i=1}^{N} H^{4}\left(0, l_{i}\right)\right] \times \mathscr{D}\left(A_{1}^{\frac{1}{2}}\right), \quad \frac{d^{2} u_{i}}{d x^{2}}\left(l_{i}\right)=0\right. \\
& \left.\frac{d^{2} u_{i}}{d x^{2}}(0)=\frac{d^{2} u_{j}}{d x^{2}}(0), \forall 1 \leq i, j \leq N, \sum_{i=1}^{N} \frac{d^{3} u_{i}}{d x^{3}}(0)=-v_{1}(0)\right\}
\end{aligned}
$$

The corresponding operator $\mathscr{G}_{d}$ is defined by

$$
\mathscr{G}_{d}\left(\begin{array}{l}
u \\
v
\end{array}\right)=\left(\begin{array}{c}
v \\
-\frac{d^{4} u}{d x^{4}}
\end{array}\right), \forall(u, v) \in \mathscr{D}\left(\mathscr{Q}_{d}\right)
$$

If $\left(u^{0}, u^{1}\right) \in X$, then the problem (3.36)-(3.40) admits a unique solution $u \in$ $C\left(0,+\infty ; \mathscr{D}\left(A_{1}^{\frac{1}{2}}\right)\right) \cap C^{1}\left(0,+\infty ; \prod_{i=1}^{N} L^{2}\left(0, l_{i}\right)\right)$ and we have: $\lim _{t \rightarrow+\infty} E(t)=0$ holds 
true for any finite energy solution of (3.36)-(3.40) if and only if

$$
\frac{l_{i}}{l_{j}} \notin \mathbb{Q}, \forall 1 \leq i \neq j \leq N
$$

Corollary 3.4. - 1. Suppose that $l_{i} / l_{j} \in \varnothing, \forall 1 \leq i \neq j \leq N$. There exists $\gamma>0$ such that for all $t \geq 0$ we have

$$
E(t) \leq \frac{C}{(t+1)^{\frac{1}{\gamma}}}\left\|\left(u^{0}, u^{1}\right)\right\|_{\mathscr{D}\left(\mathscr{G}_{d}\right)}^{2}, \quad \forall\left(u^{0}, u^{1}\right) \in \mathscr{D}\left(\mathscr{G}_{d}\right),
$$

where $C>0$ is a constant depending only on $l_{i}, i=1, \ldots, N$.

2. For all $\varepsilon>0$ there exists a set $B_{\varepsilon} \subset \mathbb{R}$, such that the Lebesgue measure of $\mathbb{R} \backslash B_{\varepsilon}$ is equal to zero, and a constant $C_{\varepsilon}>0$ for which, if $l_{i} / l_{j} \in$ $B_{\varepsilon}, \forall 1 \leq i \neq j \leq N$, then there exists $\gamma>0$ suh that for all $t \geq 0$ we have

$$
E(t) \leq \frac{C_{\varepsilon}}{(t+1)^{\frac{1}{\gamma+\varepsilon}}}\left\|\left(u^{0}, u^{1}\right)\right\|_{\mathscr{D}\left(\mathscr{C}_{d}\right)}^{2}, \quad \forall\left(u^{0}, u^{1}\right) \in \mathscr{D}\left(\mathscr{G}_{d}\right),
$$

where $C_{\varepsilon}>0$ is a constant depending only on $l_{i}, i=1, \ldots, N$ and $\varepsilon$.

Proof. - By a simple calculations we show that

$$
\lambda B_{1}^{*}\left(\lambda^{2} I+A_{1}\right)^{-1} B_{1}=\frac{i}{2 w}\left\{\frac{1}{\sum_{j=1}^{N} \operatorname{cotg}\left(w l_{j}\right)}-\frac{1}{\sum_{j=1}^{N} \operatorname{coth}\left(w l_{j}\right)}\right\},
$$

forall $\lambda=i w^{2} \in \mathbb{C}, w=r e^{i \theta}, r>0, \theta \in\left[-\frac{\pi}{2}, 0\right], \operatorname{Re} \lambda>0$.

So The condition (3.17) is satisified according to the following lemma:

Lemma 3.5. - [1, Lemma 3.3] Let $\delta>0$ be a fixed real number and $C_{\delta}=$ $\left\{w \in \mathbb{C} \mid \operatorname{Re}(w) \operatorname{Im}(w)=-\frac{\delta}{2}\right\}$. Then

$$
f(w)=\frac{i}{2 w}\left\{\frac{1}{\sum_{j=1}^{N} \operatorname{cotg}\left(w l_{j}\right)}-\frac{1}{\sum_{j=1}^{N} \operatorname{coth}\left(w l_{j}\right)}\right\}
$$

is bounded on $C_{\delta}$.

The remainder of the proof is completely similar to Corollary 3.2. 


\section{Related question}

A question related to the problem studied in this paper is a Weyl formula with second term of the same elastic networks [2].

\section{Acknowledgements}

The authors would like to thank the professor J.-P. Roth for many helpful discussions. The authors are also very grateful to the referees for their valuable comments and suggestions.

\section{Appendix}

The aim of this appendix is to prove the equality (2.1). In fact formula (2.1) can be obtained by applying Theorem 2 in [16], but for the reader convenience we give the proof. Let $G$ be a finite network made of edges $i_{l}, l=1, \ldots, N$ and $n$ vertices. We denote by $\bar{i}_{l}$ the length of $i_{l}$ and $L=\bar{i}_{1}+\cdots+\bar{i}_{N}$ the length of the graph $G$. We denote by $\mathscr{C}$ resp. $\&$ the set of edges (resp. vertices).

Fix a vertex $s$, and let $m(s)$ be the number of arcs descended from $s$. For two arcs $i$ and $j$ (containing $s$ ), we define the real number $\epsilon_{i j}=\frac{2}{m(s)}$, if $i \neq-j$ and $\epsilon_{i j}=\frac{2}{m(s)}-1$, if $i=-j$.

Consider the wave equation on the graph $G$ :

$$
\partial_{t}^{2} u(x, t)=\partial_{x}^{2} u(x, t), \partial_{t} u(x, 0)=0, u(x, 0)=f,
$$

It is well known that:

$$
u(x, t)=\sum_{C, y} \prod_{l=1}^{m} \epsilon_{i_{l} i_{l+1}} f(y), t>0 .
$$

where the sum is taken over all paths $\left(i_{1}, \ldots, i_{m}\right)$ such that $y \in i_{1}, x \in i_{m}$ with

$$
\begin{gathered}
d\left(x, I\left(i_{n}\right)\right)+\bar{i}_{2}+\cdots+\bar{i}_{m-1}+d\left(y, T\left(i_{1}\right)\right)=t, \text { for } m \geq 2, \\
d(x, y)=t \text { for } m=1 .
\end{gathered}
$$

Here $I(i)$ (resp. $T(i)$ ) is the starting (resp. end) point of the oriented arc $i$. We recall that a path $\left(i_{1}, \ldots, i_{m}\right)$ is a circuit such that $T\left(i_{1}\right)=I\left(i_{2}\right), \ldots, T\left(i_{m-1}\right)=$ $I\left(i_{m}\right)$.

For $t<0$, we have $u(x, t)=u(x,-t)$ due to the fact that $\partial_{t} u(x, 0)=0$. 
Now let $\theta \in C_{0}^{\infty}(\mathbb{R})$. A simple calculus gives

$$
\begin{aligned}
\int \theta(t) u(x, t) d t= & \int_{0}^{+\infty} \theta(t) u(x, t) d t+\int_{-\infty}^{0} \theta(t) u(x, t) d t \\
= & \frac{1}{2} \sum_{C} \prod_{l=1}^{m} \epsilon_{i_{l} i_{l+1}} \int_{i_{1}} f(y)\left(\theta \left(d\left(y, T\left(i_{1}\right)\right)+\bar{i}_{2}+\cdots\right.\right. \\
& \left.\quad+\bar{i}_{m-1}+d\left(x, I\left(i_{m}\right)\right)\right) \\
& +\theta\left(-\left(d\left(y, T\left(i_{1}\right)\right)+\bar{i}_{2}+\cdots+\bar{i}_{m-1}+d\left(x, I\left(i_{m}\right)\right)\right)\right) d y \\
& +\frac{1}{2} \sum_{i \in \mathscr{C}} \int_{i} f(y)(\theta(d(y, x))+\theta(-d(y, x))) d y .
\end{aligned}
$$

On the other hand the spectral theorem yields

$$
\int \theta(t) u(x, t) d t=\sum_{n} \iint \cos \left(\sqrt{\lambda_{n}} t\right) \theta(t) \phi_{n}(x) f(y) \phi_{n}(y) d y d t
$$

where $\left(\phi_{n}\right)_{n \geq 1}$ is an orthonormalized basis of eigenfunctions corresponding to the problem (1.4)-(1.5).

Identifying the two above equalities and integrating over $x$, we obtain

$$
\begin{gathered}
\sum_{n} \iint \cos \left(\sqrt{\lambda_{n}} t\right) \theta(t) d t=\frac{1}{2} \sum_{j \in \mathscr{C}} \theta(0)\left(\int_{j} d x+\int_{j} d x\right) \\
+\frac{1}{2} \sum_{C} \prod_{l=1, m>1}^{m} \epsilon_{i_{l} i_{l+1}} \int_{i_{1}}\left(\theta\left(d\left(x, T\left(i_{1}\right)\right)+\bar{i}_{2}+\cdots+\bar{i}_{m-1}+d\left(x, I\left(i_{m}\right)\right)\right)+\right. \\
\theta\left(-\left(d\left(x, T\left(i_{1}\right)\right)+\bar{i}_{2}+\cdots+\bar{i}_{m-1}+d\left(x, I\left(i_{m}\right)\right)\right)\right) d x .
\end{gathered}
$$

Now let $\theta \in C_{0}^{\infty}(]-\epsilon, \epsilon[)$, with $\epsilon$ small enough such that $\theta\left(\bar{i}_{l}\right)=0$ for all $l=1, \ldots, N$. In particular, we have

$$
\theta\left( \pm\left(d\left(x, T\left(i_{1}\right)\right)+\bar{i}_{2}+\cdots+\bar{i}_{m-1}+d\left(x, I\left(i_{m}\right)\right)\right)=0,\right.
$$

for all circuit $\left(i_{1}, \ldots, i_{m}\right) \neq\left(i_{l},-i_{l}\right)$. Consequently

$$
\begin{gathered}
\sum_{n} \iint \cos \left(\sqrt{\lambda_{n}} t\right) \theta(t) d t=L \theta(0)+ \\
+\frac{1}{2} \sum_{i \in \mathscr{G}} \epsilon_{-i i} \int_{i}(\theta(2 d(x, T(i))+\theta(-2 d(x, I(-i))))) d x .
\end{gathered}
$$

The assumption on the support of $\theta$ implies that

$$
\int_{i} \theta(2 d(x, T(i)))+\theta(-2 d(x, I(-i))) d x=\int_{\mathbb{R}} \theta(2 x) d x .
$$

BULLETIN DE LA SOCIÉtÉ MATHÉMATIQUE DE FRANCE 
Next, using the fact that $\epsilon_{-j j}=\frac{2}{m(s)}-1$, we obtain

$$
\begin{gathered}
\sum_{i \in \mathscr{G}} \epsilon_{-i i}=\sum_{j \in \mathscr{C}} \frac{2}{m(I(j))}-1= \\
\sum_{s \in \varnothing ; j I(j)=s} \sum_{m(s)} \frac{2}{m\left(1=\sum_{s \in \varnothing}(2-m(s))=2(n-N) .\right.}
\end{gathered}
$$

We recall that $n$ (resp. $N$ ) is the number of vertices (resp. of edges).

Summing up, we have proved that

$$
\sum_{n} \int \cos \left(\sqrt{\lambda_{n}} t\right) \theta(t) d t=L \theta(0)+\frac{n-N}{2} \int_{\mathbb{R}} \theta(x) d x .
$$

Applying the above equality to the function $e^{-i \lambda t} \theta(t)$ instead of $\theta(t)$, we obtain

Lemma 4.1. - Let $\theta \in C_{0}^{\infty}(]-\epsilon, \epsilon[)$ with $\epsilon$ small enough. We have

$$
\frac{1}{2} \sum_{n} \widehat{\theta}\left(\lambda-\sqrt{\lambda_{n}}\right)+\widehat{\theta}\left(\lambda+\sqrt{\lambda_{n}}\right)=\theta(0) L+\frac{n-N}{2} \widehat{\theta}(\lambda),
$$

for all $\lambda \in \mathbb{R}$.

\section{BIBLIOGRAPHY}

[1] K. Ammari - "Asymptotic behavior of some elastic planar networks of Bernoulli-Euler beams", Appl. Anal. 86 (2007), p. 1529-1548.

[2] K. Ammari \& M. Dimassi - "Weyl formula with second term of some elastic networks", in preparation.

[3] K. Ammari \& M. Jellouli - "Stabilization of star-shaped networks of strings", Differential Integral Equations 17 (2004), p. 1395-1410.

[4] _ "Remark on stabilization of tree-shaped networks of strings", Appl. Math. 52 (2007), p. 327-343.

[5] K. Ammari, M. Jellouli \& M. Khenissi - "Stabilization of generic trees of strings", J. Dyn. Control Syst. 11 (2005), p. 177-193.

[6] K. Ammari \& M. TucsnaK - "Stabilization of second order evolution equations by a class of unbounded feedbacks", ESAIM Control Optim. Calc. Var. 6 (2001), p. 361-386.

[7] J. VON BELOW - "Classical solvability of linear parabolic equations on networks", J. Differential Equations 72 (1988), p. 316-337.

[8] J. W. S. CASSELS - An introduction to Diophantine approximation, Cambridge Univ. Press, 1966. 
[9] R. DÁGeR \& E. ZuAZUA - Wave propagation, observation and control in 1-d flexible multi-structures, Mathématiques \& Applications (Berlin), vol. 50, Springer, 2006.

[10] B. Dekoninck \& S. NicAise - "The eigenvalue problem for networks of beams", Linear Algebra Appl. 314 (2000), p. 165-189.

[11] V. Komornik \& P. LORETI - Fourier series in control theory, Springer Monographs in Math., Springer, 2005.

[12] J. Lagnese, G. Leugerning \& E. J. P. G. Schimdt - Modelling, analysis of dynamic elastic multi-link structures, Birkhäuser, 1994.

[13] S. LANG - Introduction to diophantine approximations, Addison-Wesley Publishing Co., Reading, Mass.-London-Don Mills, Ont., 1966.

[14] S. NicAISE - "Spectre des réseaux topologiques finis", Bull. Sci. Math. 111 (1987), p. 401-413.

[15] J.-P. Roth - "Spectre du laplacien sur un graphe", C. R. Acad. Sci. Paris Sér. I Math. 296 (1983), p. 793-795.

[16] __ "Le spectre du laplacien sur un graphe", in Théorie du potentiel (Orsay, 1983), Lecture Notes in Math., vol. 1096, Springer, 1984, p. 521539.

[17] K. F. Rотн - "Rational approximations to algebraic numbers", Mathematika 2 (1955), p. 1-20; corrigendum, 168.

[18] E. J. P. G. Schmid - "On the modelling and exact controllability of networks of vibrating strings", SIAM J. Control Optim. 30 (1992), p. 229245. 